\title{
NeW APPLiCATion in MATLAB TO KNOWLEDGe TeSTING
}

\author{
Karel Perutka \& Dusan Gavenda
}
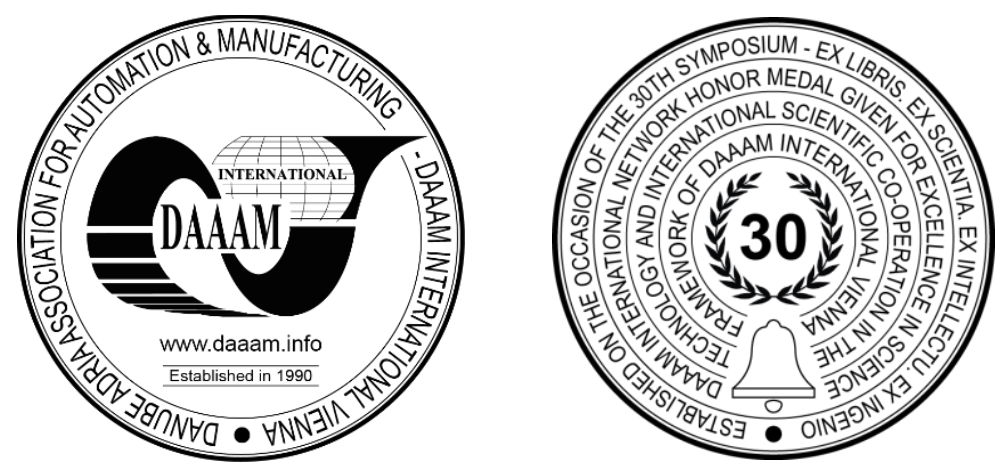

This Publication has to be referred as: Perutka, K[arel] \& Gavenda, D[usan] (2019). New Application in Matlab to Knowledge Testing, Proceedings of the 30th DAAAM International Symposium, pp.0086-0094, B. Katalinic (Ed.), Published by DAAAM International, ISBN 978-3-902734-22-8, ISSN 1726-9679, Vienna, Austria DOI: $10.2507 / 30$ th.daaam.proceedings.011

\begin{abstract}
This paper deals with the new application in MATLAB to test knowledge of students in the form of the 2D game and it was already used during teaching classes at Faculty of Applied Informatics, Tomas Bata University in Zlin, Czech Republic. The game user moves in one map, or picture, over rounded fields to the end and answers given questions about MATLAB programming loaded from the SQL database. The evaluation and analysis part of the software also exists. It gives information about the gamer type and results. Finally, the questionnaire was given to the students, and its results are also presented in the paper, together with the game and necessary preliminaries.
\end{abstract}

Keywords: Teaching aid; MATLAB; Information Didactics; Questionnaire.

\section{Introduction}

Teaching aids, applications, or in more general, new software in education, plays an important role in the teaching process during the last years. Wei and Tao [10] created nice application of multimedia aided teaching in cultural education. The advantages of multimedia-aided teaching of English were published by Guan, et al. [6]. They proved that computer multimedia-assisted English teaching could with the development of science and technology, be used widely in every aspect, especially in English teaching. Bayrak and Bayram [7] also described the increase in students' knowledge in the science and technology course. The authors applied interactive animation and simulations in chemistry.

There is also an application for teaching basic computer skills, such as the one presented by Tosun and Hatipoglu [8] which focuses on the internet aided teaching of basics computer skills for the students of the science teaching department at the faculty of education.

It is useful to compare traditional and modern teaching using a computer. The comparison was done by Aqda, et al. [9]. The authors focused their attention to the comparative effect of computer-aided instruction and traditional teaching on students ' creativity in math classes since the creativity is considered as one of the exceptional characteristics of human beings and has been the subject of many studies in different fields of education.

Virtual learning is another area where interesting solutions were realized. We can name for example the paper of Andreev, et al. [2], dealing with the education based on virtual learning robotics laboratory that is based on the analysis of data flows and functional structure of information. Moreover, educational technologies can be used for the interuniversity network, as nicely published Pryanichnikov, et al. [5]. The authors described new educational technology designed as a spatially distributed virtual network for the remote training of engineers. 
In more general, we can mention the work of Katalinic, et al. [3] about the cloud communication concept for the bionic assembly system dealing with the implementation of cloud communication in a hybrid control structure od bionic assembly system as a communication interface between subordinating and self-organizing subsystems.

There was created a new application to support teaching the MATLAB programming at the Faculty of Applied Informatics, Tomas Bata University in Zlin. It is described in the following chapters, and it is based on the work of Gavenda [1] under Perutka supervision. It continues in the work of Perutka and Zaoral [4] in different subjects and with a completely new application. Firstly, there is given the game specification describing the game description and the game rules. The description of the game follows it from the programming perspective that describes the created database and selected source code files. In the next part of the paper, the description from the user's view is presented. The final chapter shows the results of the questionnaire that evaluates the created application by students from the authors' faculty in the last year.

\section{Game specification}

\subsection{Game description}

MATLAB Quiz is a single-player practicing the knowledge of MATLAB. It is divided into five levels with increasing difficulty. Each level contains the path made up of squares that the player moves with a dice roll.

Some boxes are highlighted in blue. These boxes indicate the presence of barriers which player must stand. After stepping on the barrier window appears with the question and four possible answers from the field MATLAB, of which only one is correct. Each question has the time limit to answer. With each level, the amount of time per question decreases and the number of barriers increases.

Anyone can play MATLAB Quiz, but the player needs to have a good knowledge of MATLAB to complete the game.

\subsection{Game rules}

The goal of the game is to go through five levels. Each level contains a path consisting of boxes which are three sets of different colors. In the whole game, the player has three lives together. The yellow box indicates the player's current position. Black denotes box without special features forcing the software to do specific actions. The blue box represents the barrier. The player must answer properly to go through this part of the game. In the game, the player moves with a dice roll. If the player throws more than needed to reach the nearest barrier, he/she is asked to repeat his role. If the number fits less than necessary to reach the barrier the player moves by the appropriate number of squares. If the player reaches the barrier, a new window appears.

In this window, the image is displayed with a question and four answers and the time limit. The player must click the correct answer within the time limit. If the answer is wrong, the player gets one more question. If he/she answers wrong again, he/she loses his/her life and returns to the field. If he/she answers correctly, he/she will return to the field without losing his life. The level is completed and after reaching the last box. The game ends at the moment when the player runs out of lives, or when he completes the last level.

For each correctly answered question, players are credited by points. These points are in memory until the end of the game. There are also the additional points for the level which the player can receive, and they are stored in a database. If the result is among the top ten, then it is displayed in the Top Players table.

\section{Game from a programming perspective}

\subsection{Database}

The program uses a database in the form of an SQL server. There are three tables in this database. The question and answer table are central to the operation of the program. All questions and answers used in the ga,e are stored here, in the main part of the game. All questions are from MATLAB and were created based on our knowledge of the program.

The table consists of seven columns of which QuestionID is a primary key that is unique and automatically generated. There are two columns about the question - QuestionText and ImageUrl. The text of the question is stored directly in the image, but for better orientation it was also saved as a text in a separate column. The other columns contain the answers to the questions.

When the player runs the Game.m file on the first time, the questions are taken from the database to running software using the following command: 


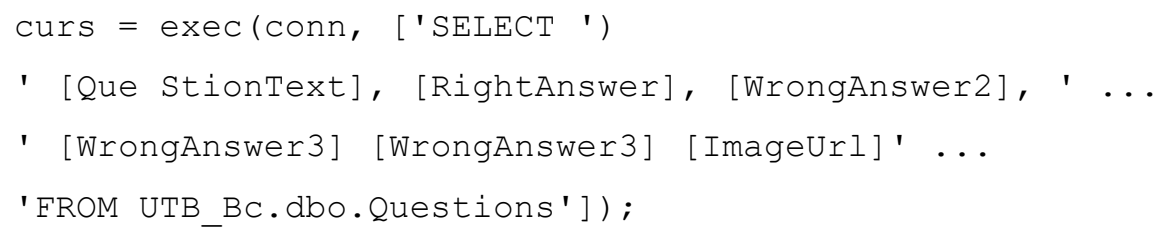

Subsequently, using the for cycle fill in the $s$ fields by objects of type Question. This field is shuffled by the randperm function. Before each new picture rendering, the possible answers are also shuffled by the function in the game. Players will never encounter the same order of questions with the correct answer on the same button.

The other two tables are linked together. The top scores table named Scores is linked to the Users table using the UserID foreign key. This connection ensures the uniqueness of each name (this logic is solved in the program).

The logic for obtaining results and players from the database is very similar to the issues of questions and answers. However, the scores and players in the database are not fixed, it is necessary to insert them. To insert it, the player must first create a table in MATLAB with the same columns as it is in the table in the database and insert the necessary data into it. It is shown in the syntax below.

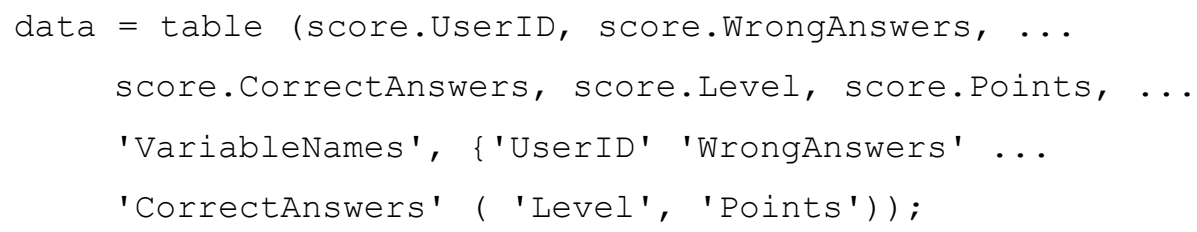

This data can then be written to the database using the sqlwrite command.

\subsection{Description of selected source code files}

The application consists of three FIG files and seventeen $M$ files. Each FIG file is linked with $M$ file of the same name, these files form the core of the entire application, the other files realize the support functions and are classified according to their use. The largest group of files are database-based files in the SQLScripts directory. Data that are sent or received from the database are stored in objects created by class type, these files are stored in the Models directory.

All three files, Question.m, Score.m, and User.m, serve as object creation classes. Each file represents a table of the same name in the database and contains several properties that copy the columns in the tables.

The OpenDBConnection.m file is a file with a single function of the same name. It was created to minimize the repetitive code. The code that is stored in this function, is required whenever the database is used, so therefore it should be repeated in several other files. This file fixes this. The very task of this file is to set up and establish a connection to the database. The CloseDBConnection.m file was created for the similar reason as OpenDBConnection.m. The task of this file is to close the database connection and clean up the created variables.

The main GetQuestions function from GetQuestions.m file calls function to establish a connection, retrieve data, and close a database connection. Subsequently, it populates the object class Question by obtained data and fields are stirred using the code:

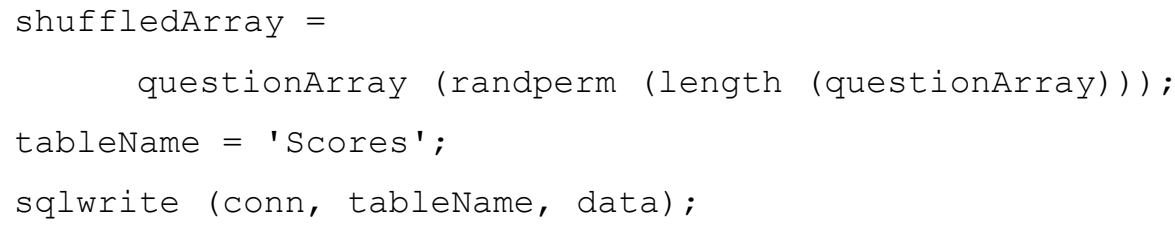

File GetScores.m works similarly to the GetQuestions.m file. The main GetScores function calls the get ScoresFromDatabase function to get the top results from the database and then populates the array of Score objects with those results. 
At first glance, the GetUsers.m file has the same function as other files starting with Get. However, after obtaining data from the database, it is needed to verify whether there already exists the name of the player who just played. If so, its information is returned to the program. If it is not already in the database, the SaveUser function is called.

The SaveUser function in the SaveUser.m file in the first section creates a table into which the record with the player's name is inserted, then calls the saveUserToDatabase function to execute the save itself. However, it then returns the ID that was generated when it was saved and returned to the SaveUser function. A User object is created to continue this function where ID and name are assigned from the database.

File Мепи.m is the default file of the whole application, from another file the application fails to run properly because all files have dependencies and require parameters without which they cannot run.

\begin{tabular}{|lll|}
\hline Function name & Position in the file & Purpose \\
\hline Menu_OpeningFcn & $23-35$ & $\begin{array}{l}\text { Setting name, clean the variables } \\
\text { from previous games calling }\end{array}$ \\
\hline Rules Button_Callback & $44-48$ & Hide menu elements and display rule elements \\
\hline leaderboardButton_Callback & $51-75$ & Hiding menu elements, retrieving data from \\
\hline the database, and displaying the top player's \\
\hline returnButton_Callback
\end{tabular}

Table 1. Menu.m file functions

The Menu_OpeningFcn function runs every time the user runs the application. This file has to execute commands that must be executed before the user can do anything. In this case, the centering of the window in the middle of the screen, setting the title and, mostly, clear all global variables. Deleting global variables prevents application crashes due to bad values of variables.

Another function is callback of rulesButton button, i.e. button for displaying game rules. This function is only hiding all the features menu using hideMenu function, displays the text of the rules and Back button.

On the same principle as a leaderboardButton_Callback function works. This time, however, its purpose is not only to display high scores, but also to call an extra function from GetScores.m file to get the best results from the database. These data are processed into a form that can be viewed using a spreadsheet.

The playButton_Callback function is simply a redirection to the MiniGame.m file. Functions hideMenu and showMenu performs exactly the opposite function and exists to minimize the repetitive code. At least one of them is used when almost all buttons are pressed. ReturnButton_Callback functions perform the opposite action of the rules and best results, hiding the elements that made these functions visible and showing the elements that were hidden. 
The MiniGame_OpeningFcn function takes care of adjusting the visibility and position of axes elements in which boxes and cube are drawn. Also, the initial level, hit points are initialized, and the setPositionAndPrepareBarriers function is called. Another important function is to verify that the player has reached the last box, in these circumstances the SetScore function is called (if the player is in the fourth level) or the level is raised. Also, the functions for drawCircles and setLivesCount are called here.

ThrowButton_Callback function is called at the moment the user presses a Throw the dice button. First, a random integer from one to six is generated using the randi function. Furthermore, the function calls setThrownNumberImage unique function to render cubes based on the generated number from one to six.

The drawCircles function is used to render the fields. In this function, there is a cycle to render 25 frames whose positions are saved in the field. In each pass through the cycle, it displays one box, in which apart from changing position it also changes its color. It based on the contents of the variable CurrentPosition (determines the current position of the player) and field barrierIndexes (generated positions of barriers). Sample code is shown below

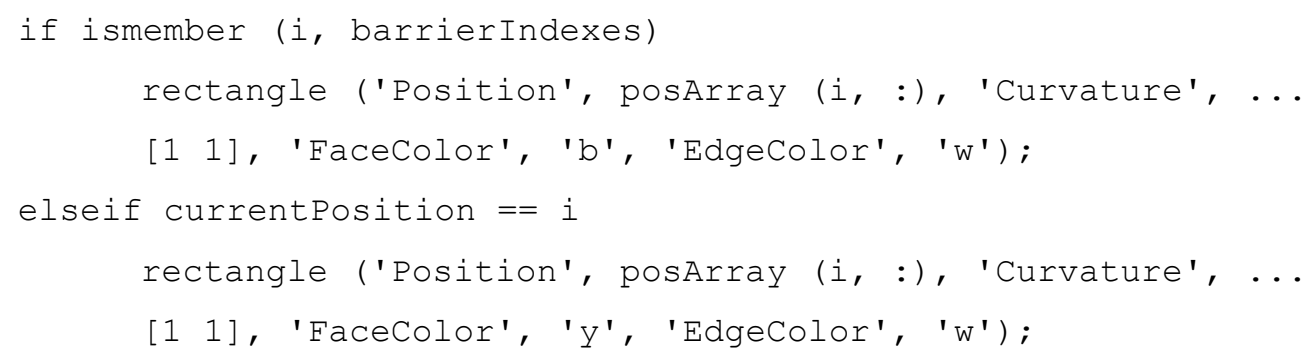

Barriers are generated in the prepareBarriers function, which uses the randperm function to generate an array of barriers, the amount of which varies according to the level.

Function advanceToNextPosition is an easy way to clear the path. The main part of the function is while cycle, which increases the player's current position in each pass and uses the drawCircles function to redraw the boxes with the new player position. This cycle ends when the player reaches a position where the desired position equals the player's current position. It is described by the code below

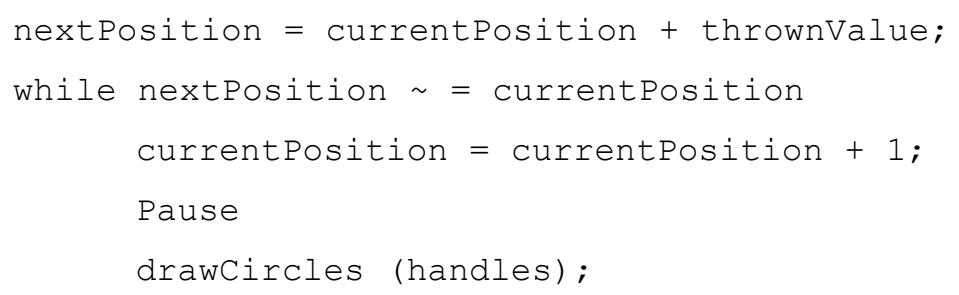

Two simple functions follow. The first one is setPositionAndPrepareBarriers, which is called when starting the program from the MiniGame_OpeningFcn function and is set to set the player's position to 1 and call the prepareBarriers function.

The last function in the file MiniGame.m is setThrownNumberImage function that works similarly as drawCircles function, but it does not render the box, but the dice with the thrown number. Deciding on the number of dots on the cube is solved using a switch with six variants. Each of these variants creates a field with different dots positions on the cube. 


\begin{tabular}{|l|ll|}
\hline Function name & Position in the file & Purpose \\
\hline MiniGame_OpeningFcn & $23-62$ & Setting variables and GUI elements \\
\hline throwButton_Callback & $77-123$ & $\begin{array}{l}\text { Generating thrown numbers and comparing } \\
\text { the position with the position of the further } \\
\text { barrier }\end{array}$ \\
\hline drawCircles & $126-158$ & Rendering of boxes \\
\hline prepareBarriers & $161-174$ & Generation of barrier indices \\
\hline advanceToNextPosition & $177-190$ & Move through the boxes \\
\hline setPositionAndPrepareBarriers & $193-198$ & $\begin{array}{l}\text { Initial setup position and call prepareBarriers } \\
\text { function }\end{array}$ \\
\hline setLivesCount & $201-205$ & Display the number of lives \\
\hline setThrownNumberImage & $208-242$ & Render cubes \\
\hline
\end{tabular}

Table 2. MiniGame.m file functions

\section{Application from the user's view}

\subsection{Main menu}

The user can run the application using the Menu command from MATLAB Command Window. After launching the application, the user sees the main menu dialog of the game (see Fig. 1 in the form of a menu with four items. Each item is handled by a button that the user can press by moving the cursor on it and pressing the left mouse button. The user is redirected to the screen with the recorded table of the best players when pressing the Best players button $\left(2^{\text {nd }}\right.$ one). This table contains four columns, and is shown up to 10 entries sorted according to highest scores. The only button on this screen is the Back button, which returns the user to the menu. Using the Rules button ( $3^{\text {rd }}$ one) users can read the game rules. On this screen, the user has only one button, the Back button, which brings him back to the main menu.

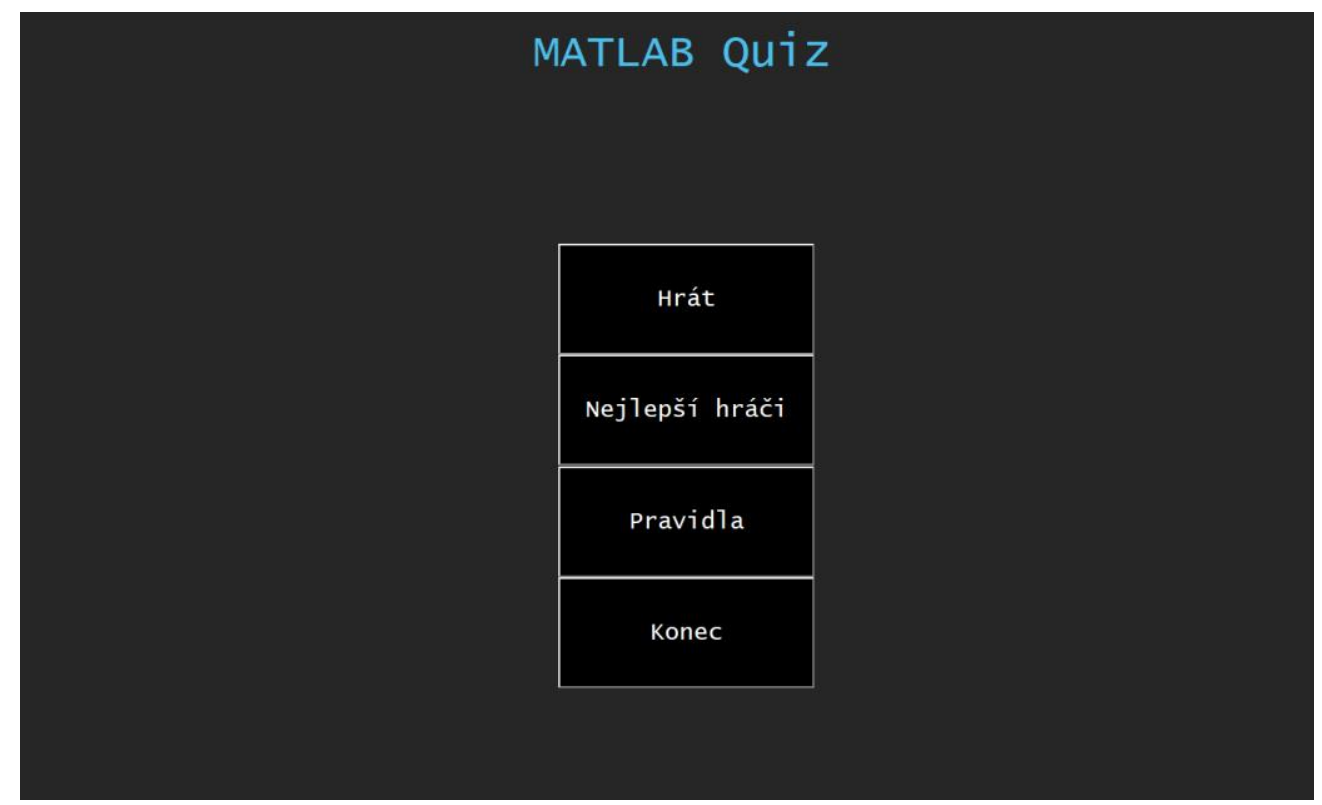

Fig. 1. Main menu dialog of the game 
Pressing the Play button ( $1^{\text {st }}$ one) will turn off the current GUI and start a new one. On the new screen, a player can see twenty-five boxes with different colors on the left side (see Fig. 2.). The properties of these boxes are explained in the game rules dialog. At the right edge of the window, the player sees two buttons and two information lines.

Here the player learns at what level and how many lives he/she has left. Using Finish the game button, the player can once again return to the menu.

Throw the dice button may cause several events. Each time the player press this button, the cube is drawn with the number of dots that representing what number is thrown. If the number is greater than the distance of the nearest barrier, a new text box will appear prompting the user to roll again. If the fit is less than the distance to the nearest barrier, the player begins to move forward over the fields, or circles gradually. If the player's position is on the barrier, the current window is turned off and a new window opens, see Fig. 3.

In the new window, the user sees an image with a question (that may or may not relate with the displayed picture) and four buttons with answers. As in the previous window, the End Game button appears. What's new in this window? It is the Time Remaining text box that tells the user how much time he/she has left to answer a question.

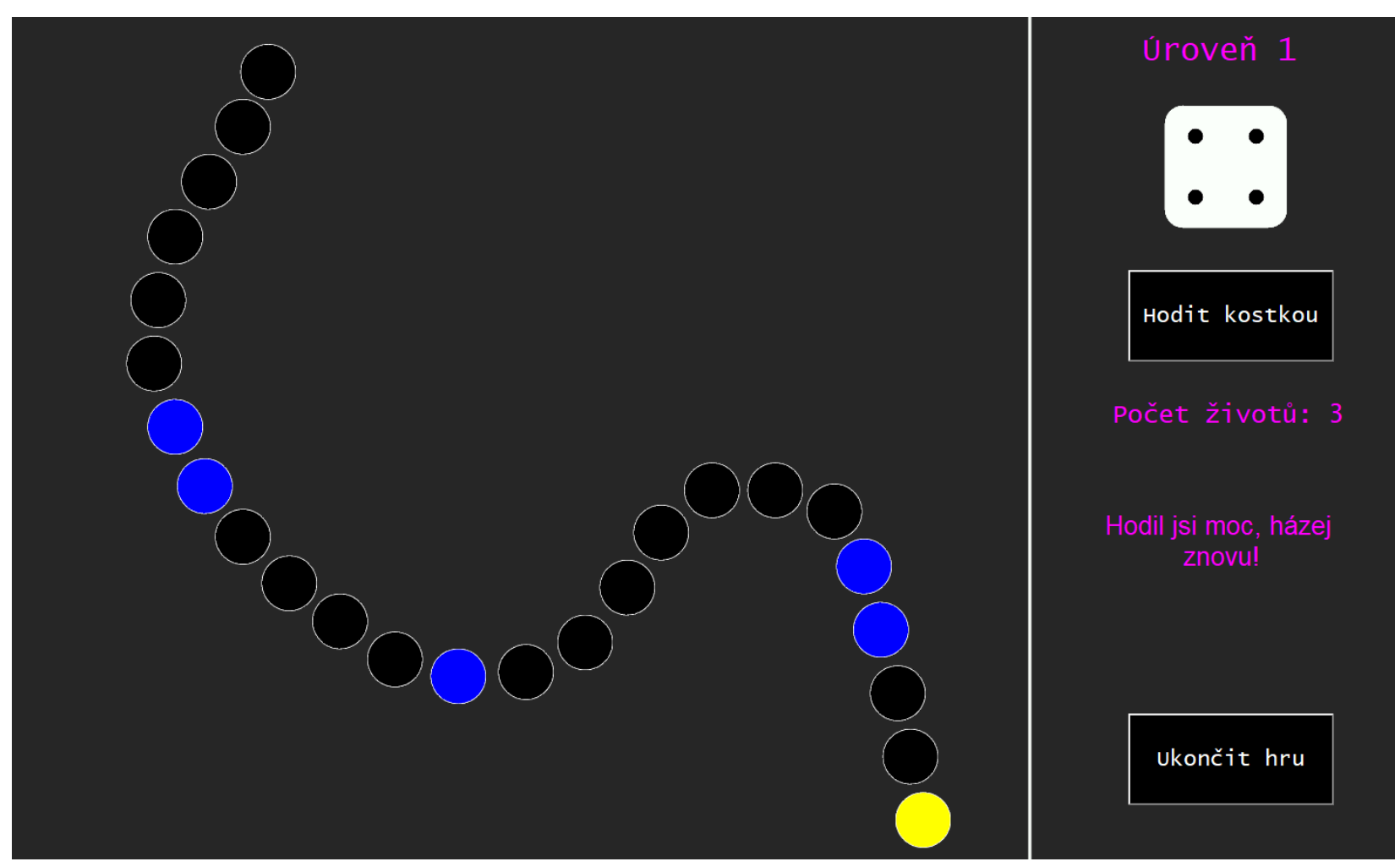

Fig. 2. Main player window

If the player fails to answer the question within the time limit, the question is evaluated as being incorrectly answered. If the answer is wrong or time runs out, he/she gets an extra question, the time limit returns to its original value. If he/she fails to respond correctly again, he/she loses one life, the window closes, and the previous box with the boxes opens again, and the user has now taken the position of the barrier. In the case the player answers the question correctly and in a time limit, he/she also returns to the previous screen, now with the same number of lives as before.

By reaching the last field, the user invokes the information dialog, after clicking on this dialogue, the player is moved to the next level. This time, the player has got through more barriers and he/she has less time to answer the questions.

If the user gets up to the final level and manages to go through all the barriers and reaches the last frame, the dialog box is displayed, indicating the number of correct and bad answers, and also the number of points achieved. Clicking on the $O K$ button displays another dialog. This time the user is asked to fill in his name. If the user does not write anything and clicks the $O K$ or Cancel button, the game still recognizes the player and stores the new record under No Name text. This information is then stored in a database and is shown in the table of the best result, if the number of reached points is in the top ten. After closing the dialog for filling in the player's name, the main menu dialog is opened again. 


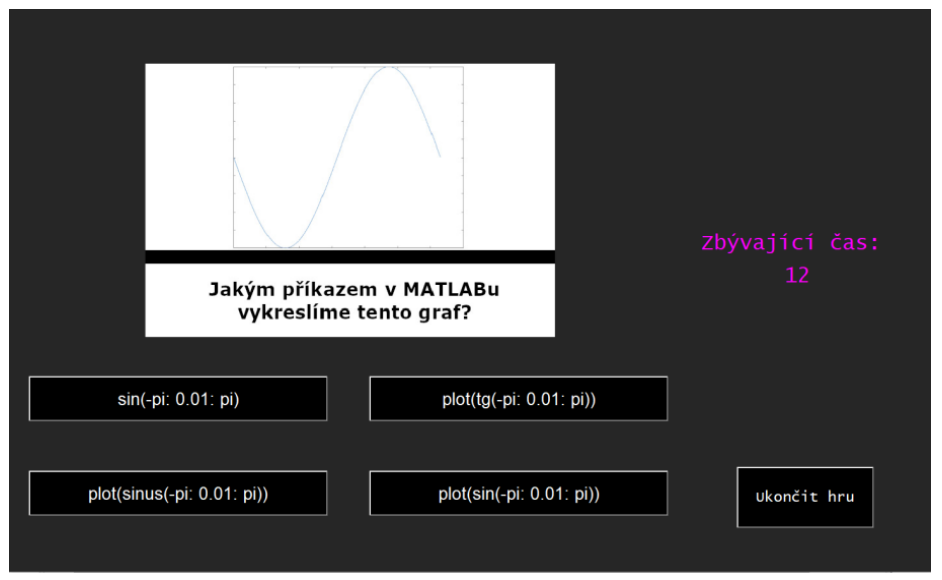

Fig. 3. Sample window with the question

\section{Evaluation using Questionnaire}

The following questionnaire was created in purpose to evaluate the created application by students of regular and distance curricula. The questions were the following ones:

1) Have you used the created application to practice your knowledge?

2) Have you reached the end of the final level?

3) Should this application have more levels?

4) Do you have a similar application in some other subject of your curriculum?

The obtained results are displayed below.
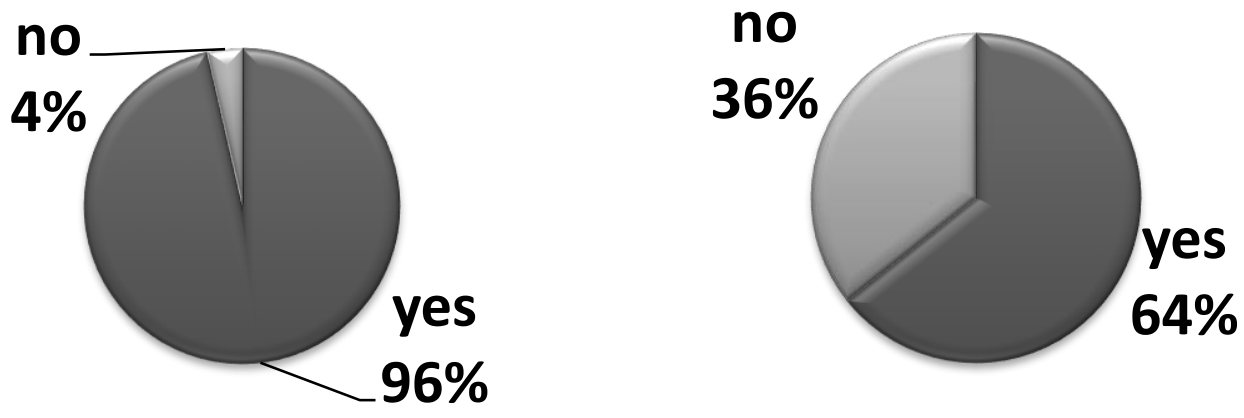

Fig. 4. Answers on Question 1 (left) and Question 2 (right)
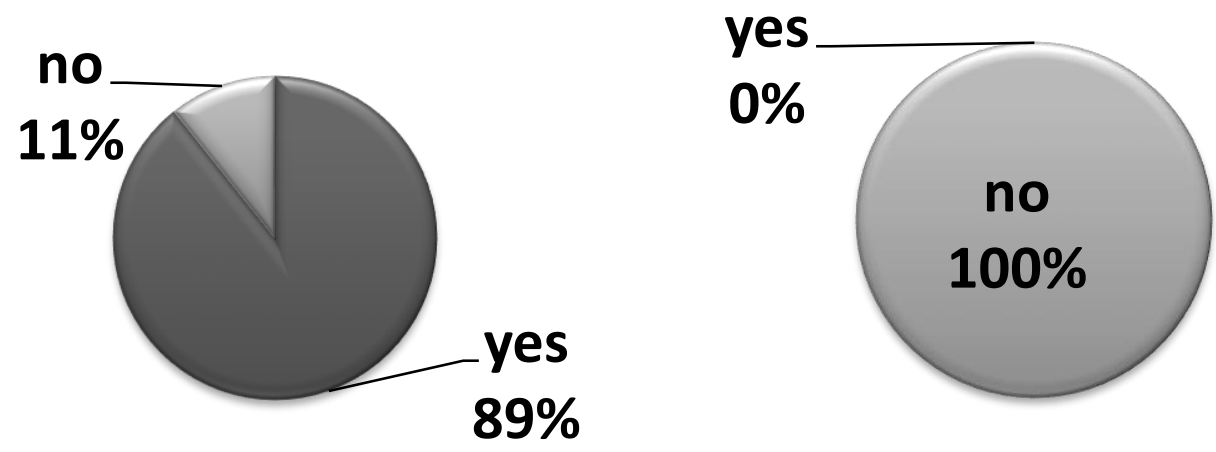

Fig. 5. Answers on Question 3 (left) and Question 4 (right)

It is clear from the presented results of the questionnaire and the graphs in figures 4 and 5 that in general students very positively evaluated created application. Some of them, however, did not reach the last field of the game. Moreover, they would like to have more levels to practice their knowledge and all of them confirmed that there is no similar software in the other subject in their curriculum. 


\section{Evaluation of the created solution}

The created application became the part of new modern educational aids according to the modern strategy of teaching that implements the lectures and practices in the attractive form. Games are leading applications in this area. The students confirmed that they are more attracted to the studies. The tests that verified the knowledge of students from the area of MATLAB programming confirmed the better knowledge of students who played the game in comparison to those who did not use this game.

The issue of the paper was to solve the problem - to find something to increase the knowledge of MATLAB programming at the faculty in the given curricula. It is important to understand the game functionality, and it is also important to have the knowledge of MATLAB in purpose to finish the created game. The contents of the game are now limited to practice and test the knowledge of MATLAB programming now.

\section{Limitations and plans}

Although the paper has reached its aims, there were some limitations. First, because the game is new, it was verified only by a small group of students who were attending the classes. Therefore, it is necessary to use it for some years to generalize the result and to find what to improve and enlarge. Second, the content of the game is specific. It was created for the given curriculum, so it is necessary to modify or enlarge it on purpose to use this game by users from another curriculum and other faculty.

Future work is divided into several parts. Firstly, it is necessary to use the game for more years in purpose to evaluate the game from the users' point of view. Lately, there will be realized necessary correction based on the experience with this application.

\section{Conclusion}

The necessity to attract the study curricula about intelligent systems with robotics was the main problem that had to be solved. It was solved by a modern approach using the game as the idea of teaching aid. It leads to newly created application practicing the student knowledge of MATLAB programming using the quiz. This application was positively evaluated by students. Students used it before the final test from automation in the selected subjects. It covers the whole introductive course to MATLAB and examples on how to solve them. Future work will be oriented on the more complex enlargement of the newly created application. The enlargement will be focused on the higher increase in the number of topics and questions in the database and on the implementation in more subjects of the existing curricula.

\section{References}

[1] Gavenda, T. (2019). The Development of an Environment for Interactive Applications in MATLAB, Thesis, Faculty of Applied Informatics, Tomas Bata University in Zlin, Zlin, Czech Republic

[2] Andreev, V.; Kuvshinov, S.; Pryanichnikov, V. \& Poduraev, Y. (2014). Education on the Basis of Virtual Learning Robotics Laboratory and Group-Controlled Robots. Procedia Engineering, Vol. 69, pp. 35-40, ISSN 1877-7058

[3] Katalinic, B.; Kukushkin, I.; Pryanichnikov, V., \& Haskovic, D. (2014). Cloud Communication Concept for Bionic Assembly System. Procedia Engineering, Vol. 69, pp. 1562-1568, ISSN 1877-7058

[4] Perutka, K. \& Zaoral, T. (2015). Multimedia Teaching Aid for Students of Basics of Control Theory in MATLAB and SIMULINK. Procedia Engineering, Vol. 100, pp. 150-158, ISSN 1877-7058

[5] Pryanichnikov, V.E.; Katalinic, B.; Kirilchenko, A.A., Khelemendik, R.V., Kuvshinov, S.V.; Vician, D. \& Uglesic, A. (2015). New Creative Educational Technologies for Inter-University Network. Procedia Engineering, Vol. 100, pp. 259-268, ISSN $1877-7058$

[6] Guan, N.; Song, J. \& Li, D. (2018). On the Advantages of Computer Multimedia-aided English Teaching. Procedia Computer Science, Vol. 131, pp. 727-732, ISSN 1877-0509

[7] Bayrak, B.K. \& Bayram, H. (2010). The effect of computer aided teaching method on the students“ academic achievement in the science and technology course. Procedia Social and Behavioral Science, Vol. 9, pp. 235-238, ISSN 1877-0428

[8] Tosun, N. \& Hatipoglu, N. (2009). Internet aided teaching of basic computers skills for the students of science teaching department from the faculty of education in Trakya University. Procedia Social and Behavioral Science, Vol. 1, pp. 105-111, ISSN 1877-0428

[9] Aqda, M.F.; Hamidi, F. \& Rahimi, M. (2011). The comparative effect of computer-aided instruction and traditional teaching on student's creativity in math classes. Procedia Computer Science, Vol. 3, pp. 266-270, ISSN 1877-0509

[10] Wei, C. \& Tao, Y. (2012). Application of Multimedia-aided Project-teaching Mode in Cultural Education. Procedia, Vol. 2, pp. 538-542, ISSN 2212-6678 